Reprod. Nutr. Dévelop. 1980, 20 (2), 485-498.

\title{
Aspects génétiques des arrêts précoces du développement.
}

\author{
par A. BOUÉ, Joëlle BOUÉ, P. COUILLIN \\ Groupe de Recherches de Biologie Prénatale, INSERM U. 73 \\ Château de Longchamp, Carrefour de Longchamp, Bois de Boulogne, 75016 Paris.
}

Summary. The genetic aspects of prenatal wastage.

Recent surveys have provided more detailed data on the frequency of chromosome aberrations in prenatal wastage. In large surveys, the abnormality rate of spontaneous abortions is 50 to 60 p. 100 and, except for mosaicism, the relative frequencies of the different types of chromosome aberrations are usually similar. The frequency of mosaicism is higher in some studies done in the U.S. and very much lower in most European reports; the methods used cannot explain this discrepancy.

The exact identification of the extra chromosome in autosomal trisomy supplies data on the time of developmental arrest in relation to the types of trisomy. This might explain the wide variations in the frequencies of different autosomal trisomies since studies on spontaneous abortions only include abortuses having a developmental age of three or more weeks.

Using various approaches (chromosome markers, HLA typing), the mechanisms of some chromosome numerical errors have been determined ; triploidy with two paternal sets of chromosomes, especially that resulting from dispermy, was the most frequent $(50$ to 70 p. 100). HLA typing of couples who had a triploid conceptus showed an increase in the frequency of parents having two different antigens in common, one in each locus; this might explain dispermy.

Structural chromosome aberrations represent 4 to 6 p. 100 of chromosomally abnormal abortuses. In our series, 39 of those aberrations were observed, including 23 unbalanced robertsonian translocations, 12 of which were inherited (11 of maternal origin). This situation was similar to the observations of unbalanced robertsonian translocations at birth.

Other than chromosomal aberrations, the genetic causes of prenatal wastage have been demonstrated rarely in humans. An observation of four generations of familial agenesia of the sacrum associated or not with spina bifida showed a linkage with genes localized on the short arm of chromosome 6 (HLA); this would suggest that situations similar to the $T$ locus in mice may exist in humans.

C'est surtout au cours des quinze dernières années que la grande fréquence des arrêts du développement du produit de conception avant terme a pu être appréciée chez l'homme. Dans l'analyse de ce « déchet prénatal » (prenatal wastage) deux questions principales se posent :

1. comment évaluer aux différents stades du développement l'importance du déchet prénatal par rapport à l'ensemble des fécondations ? 
2. quelle est la part de responsabilité des causes génétiques?

Dans l'espèce humaine une évaluation rigoureuse des arrêts du développement se heurte à des obstacles difficilement surmontables : critères de la fécondation, collecte des « zygotes 》 expulsés... Mais on est frappé par le fait que des évaluations obtenues par des approches indirectes très variées conduisent toutes à penser que le déchet prénatal est égal ou supérieur à $50 \mathrm{p}$. 100 des conceptions. Leridon (1977) a établi à partir des données de deux importantes études (French et Bierman, 1962 ef Hertig et Rock, 1959) une telle évaluation (tabl. 1).

Les travaux des dernières années ont surtout porté sur l'analyse des avortements spontanés précoces (en pratique essentiellement le premier trimestre de la gestation) ef de la mortalité périnatale (morts in utéro, mort-nés, morts dans la première semaine), les techniques d'analyse ont été la description anatomo-pathologique, macroscopique et microscopique, et l'analyse chromosomique. Dans ces travaux, outre le recueil des spécimens, les difficultés d'analyse ont été dues à l'état fréquent de macération lié à la rétention in utero.

Une grande lacune persiste, c'est l'analyse des arrêts très précoces du développement, en pratique les deux premières semaines suivant la fécondation ; l'ignorance d'une fécondation, l'impossibilité du recueil des produits expulsés, rendent vaines toutes tentatives d'étude directe. On sait pourtant que ces arrêts très précoces existent, soit par des études hormonales systématiques au cours du cycle, soit par des études anatomiques, ainsi Philippe (1974) a observé dans des biopsies d'endomètre des œufs anormaux implantés et arrêtés à 10-12 jours de développement.

Peut-on à partir des études chromosomiques sur les avortements spontanés précoces faire une extrapolation vers les premiers stades du développement?

\section{TABLEAU 1}

Table complète de mortalité intra-utérine (pour 100 ovules exposés au risque de fécondation) (d'après les résultats de Hertig et de French et Bierman)

\begin{tabular}{|c|c|c|}
\hline $\begin{array}{l}\text { Semaine après } \\
\text { l'ovulation }\end{array}$ & Décès $\left({ }^{1}\right)$ & Survivants $\left({ }^{2}\right)$ \\
\hline & $16(3)$ & 100 \\
\hline 0 & 15 & 84 \\
\hline 1 & 27 & 69 \\
\hline 2 & 5,0 & 42 \\
\hline 6 & 2,9 & 37 \\
\hline 10 & 1,7 & 34,1 \\
\hline 14 & 0.5 & 32,4 \\
\hline 18 & 0,3 & 31,9 \\
\hline 22 & 0,1 & 31,6 \\
\hline 26 & 0,1 & 31,5 \\
\hline 30 & 0,1 & 31,4 \\
\hline 34 & 0,1 & 31,3 \\
\hline 38 & 0,2 & 31,2 \\
\hline Nés vivants. & & 31,2 \\
\hline
\end{tabular}

(1) Plus précisément, expulsions d'embryons décédés.

(2) Grossesses encore en cours.

(3) Echecs à la fécondation. 
Plusieurs études importantes sur les avortements précoces conduisent à évaluer la fréquence des aberrations chromosomiques aux environs de 50 et 60 p. 100 . Dans la plupart des études cetle fréquence est calculée en fonction de l'âge gestationnel, c'est-à-dire l'âge de la grossesse au moment de l'expulsion du produit d'avortement. La courbe de Creasy et al. (1976) (fig. 1) en est un exemple, des courbes superposables ont été obtenues par Kajii (1979) et par Warburton (1978). On voit que plus on remonte vers la dixième semaine gestationnelle plus augmente la fréquence des anomalies chromosomiques. Mais dans ces trois études on note une diminution de la fréquence des anomalies chromosomiques lorsque l'âge gestationnel se situe entre 8 et 10 semaines. On doit d'abord remarquer que le nombre de spécimens recueillis à ce moment est réduit, mais surtout - comme le remarque Kajii - on peut se demander s'il n'existe pas alors un biais de sélection qui conduirait à éliminer les produits avec anomalie chromosomique.

FIG. 1. - Fréquence des anomalies chromosomiques en fonction de la date d'expulsion de l'abortus (Creasy ef al., 1976).

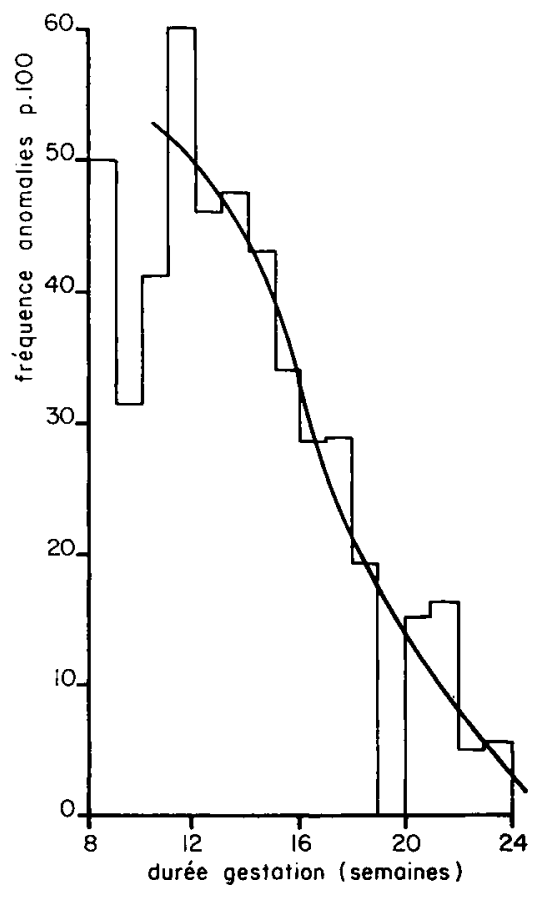

En effet, une des caractéristiques essentielles des avortements dus à une anomalie chromosomique est la longue rétention in ufero après l'arrêt du développement, le système hormonal maternel continue sa fonction quelque temps et maintient la grossesse quelques semaines avant que l'expulsion se produise.

La figure 2 montre, dans le cas des trisomies autosomiques (Boué ef al., 1976), la comparaison entre la durée du développement atteint par le zygote ef l'âge gestationnel auquel s'est produite l'expulsion. On voit que bien que les arrêts au cours de la troisième semaine du développement soient très fréquents, les expulsions avant la dixième semaine gestationnelle sont rares. Si une anomalie chromosomique est responsable d'un arrêt du développement très précoce l'œuf peut ne pas être recueilli 
ou ne pas être identifié parmi les produits expulsés, par contre, s'il s'agit d'une cause maternelle, le développement de l'œuf correspond à l'âge gestationnel ef il sera ainsi plus facile à recueillir et à identifier.

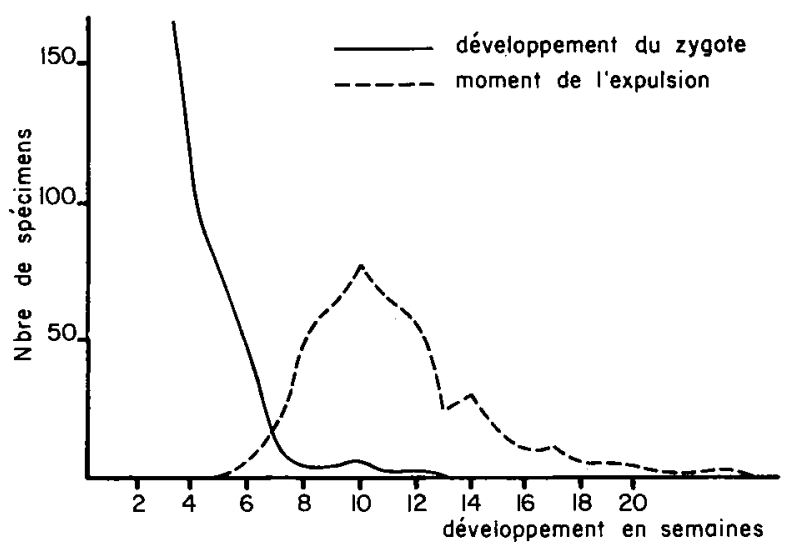

FIG. 2. - Durées de développement des zygotes et durées de la gestation dans les trisomies autosomiques létales (Boué et al., 1975).

Ces raisons nous ont conduits à préférer les évaluations de la fréquence des anomalies chromosomiques en fonction du développement du zygote, durée déterminée sur l'examen macroscopique ef microscopique du placenta et de l'embryon (Boué et al., 1976).

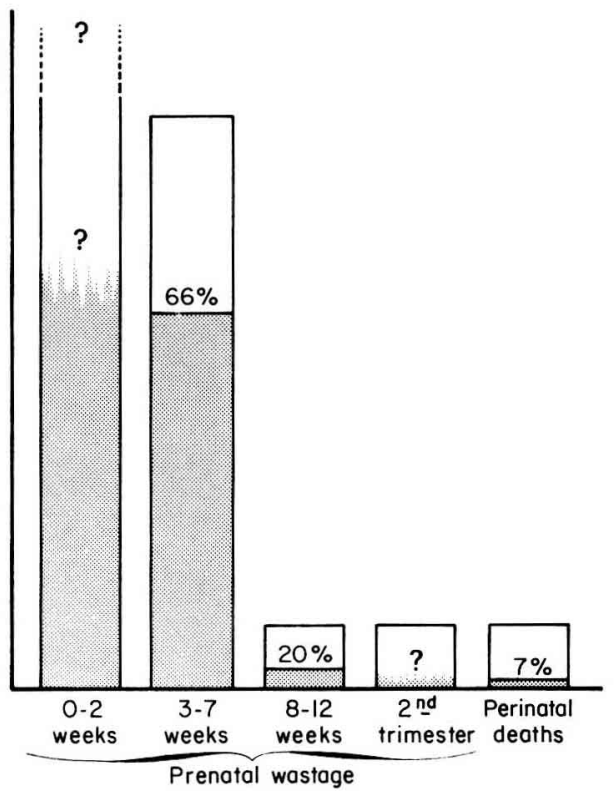

FIG. 3. - Fréquences relatives des arrêts du développement et pourcentage des anomalies chromosomiques oux différents stades de développement du zygote. 
A partir des données provenant des études sur les avortements spontanés et sur la mortalité périnatale (Sutherland et al., 1978) on peut tenter d'établir une représentation de la fréquence des arrêts du développement el du pourcentage des anomalies chromosomiques en fonction des stades du développement (fig. 3). On possède dès maintenant des informations précises sur les arrêts du développement de 3 à 12 semaines et sur la période périnatale, la fréquence des arrêts après la douzième semaine peut être appréciée (1 à 2 p. 100 des avortements cliniques), mais on ne dispose pas de résultats d'analyses chromosomiques systématiques, on peut situer l'incidence des anomalies entre 20 p. 100 (chiffre observé à 20 semaines) et 7 p. 100 (chiffre observé dans la mortalité périnatale). Beaucoup plus difficiles sont les évaluations concernant les deux premières semaines du développement. Grâce à des dosages d'HCG entrepris systématiquement au cours de cycles menstruels chez des femmes désirant un enfant, on a pu déceler des arrêts très précoces. Ainsi (Williamson et Miller, 1979) sur 148 fécondations décelées par dosages d'HCG, seules 87 (58,4 p. 100) ont éłé menées à terme, 47 (31,7 p. 100) n'ont été reconnues que biochimiquement et 14 ont conduit à un avortement spontané cliniquement décelable. Il est probable que s'il était possible de déceler les fécondations avant l'implantation on obtiendrait des chiffres encore plus élevés. La fréquence des anomalies chromosomiques responsables de ces arrêts précoces ne peut évidemment pas être évaluée.

Quelles sont les anomalies chromosomiques observées dans les avortements spontanés?

Les résultats sur les fréquences relatives des diverses aberrations sont remarquablement similaires dans toutes les séries (tabl. 2). Quelques précisions ont été obtenues ces dernières années sur certaines anomalies.

TABLEAU 2

Fréquence des différents types d'anomalies chromosomiques dans les avortements Résultats d'Etudes Européennes (Danemark, France, Allemagne, Suisse, Royaume-Uni)

Monosomie $X \ldots \ldots \ldots \ldots \ldots \ldots \ldots \ldots$
Trisomies $\ldots \ldots \ldots \ldots \ldots \ldots \ldots \ldots \ldots \ldots$
Triploïdie $\ldots \ldots \ldots \ldots \ldots \ldots \ldots \ldots \ldots \ldots$
Tétraploïdie $\ldots \ldots \ldots \ldots \ldots \ldots \ldots \ldots \ldots$
Anomalies de la structure $\ldots \ldots \ldots \ldots$
Mosaiques $\ldots \ldots \ldots \ldots \ldots \ldots \ldots \ldots \ldots$

\section{1. - Les trisomies autosomiques (tabl. 3).}

A partir de 9 séries importantes (Boué et al., 1976 ; Carr el Gedeon, 1978 ; Creasy et al., 1976 ; Dill, 1978 ; Hassold et al., 1977 ; Kajii et al., 1979 ; Lauritsen, 1976 ; Takahara, 1978 ; Warburton, 1978) nous avons réuni 625 observations de trisomies autosomiques identifiées par les techniques de bandes. Plusieurs remarques peuvent être formulées : 
- A l'exception du chromosome 1, tous les autosomes ont été impliqués dans des trisomies responsables d'avortements spontanés.

- De très grandes variations sont observées dans la fréquence relative de ces diverses trisomies. On est d'abord frappé par l'extraordinaire fréquence de la trisomie 16 qui, à elle seule, représente un tiers des trisomies. Ce sont, avec la monosomie $X$, les deux aberrations chromosomiques les plus fréquemment identifiées. A l'opposé certaines trisomies sont exceptionnelles.

- On remarque aussi que dans le groupe D d'une part et dans le groupe G d'autre part, les diverses trisomies sont d'importance équivalente.

\section{TABLEAU 3}

Trisomies autosomiques dans les avortements spontanés

Séries de Boué, Carr, Creasy, Dill, Hassold, Kajii, Takahara, Therkelsen, Warburton

\begin{tabular}{rrrrrr}
\hline Trisomie & Nb & p. 100 & Trisomie & Nb & P. 100 \\
\hline 1 & & & 13 & 33 & 5,3 \\
2 & 28 & 4,5 & 14 & 41 & 6,6 \\
3 & 5 & & 15 & 46 & 7,4 \\
4 & 11 & 1,8 & 16 & 206 & 33,0 \\
5 & 1 & & 17 & 1 & \\
6 & 3 & 18 & 32 & 5,1 \\
7 & 24 & 3,8 & 19 & 1 & 1,9 \\
8 & 25 & 4,0 & 20 & 12 & 8,8 \\
9 & 20 & 3,2 & 22 & 55 & 9,9 \\
10 & 14 & 2,2 & & 62 & \\
11 & 1 & & Total & 625 & \\
12 & 4 & & & & \\
\hline
\end{tabular}

Une question se pose : les fréquences relatives observées représentent-elles l'incidence de ces trisomies au moment de la fécondation ? Ou, au contraire, sont-elles le reflet d'un biais de sélecrion dans les spécimens recueillis, lié aux différences dans le développement compatible avec chacune de ces trisomies? Les travaux de Gropp et al. (1976, 1979) sur la souris mus poschiavinus (tobacco mouse) ont montré que pour chaque irisomie autosomique il existe un stade précis d'arrêt du développement. En est-il de même dans l'espèce humaine?

Nous avons évalué la durée du développement des diverses trisomies autosomiques et pour quelques-unes le nombre des spécimens étudiés a permis de préciser la durée du développement.

La figure 4 montre les durées de développement dans deux groupes de trisomies : trisomies $E$ ef trisomies G. Dans le groupe E l'arrêt du développement se situe à 3 semaines pour les trisomies 16 , à 6 semaines et plus pour les trisomies 18 , qui peuvent également aller à terme, par contre la trisomie 17 n'a pratiquement jamais été recueil- 
lie. Dans le groupe G l'arrêt du développement se situe autour de 4 semaines pour la trisomie 22 et à 6 semaines et plus pour la trisomie 21 .

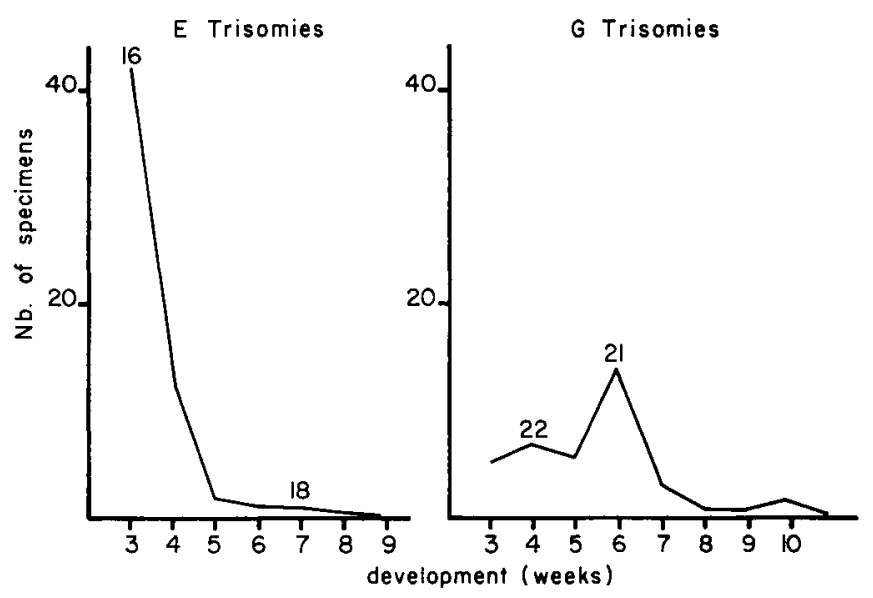

FIG. 4. - Durées du développement dans les trisomies $E$ ef les trisomies $G$ létales (Boué et al., 1976).

On peut penser qu'il existe pour chaque trisomie autosomique une courbe figurant les durées du développement compatibles avec cette anomalie et la position de cette courbe par rapport aux possibilités de recueil des prélèvements peut expliquer les variations dans la fréquence des anomalies observées dans les avortements spontanés (fig. 5).
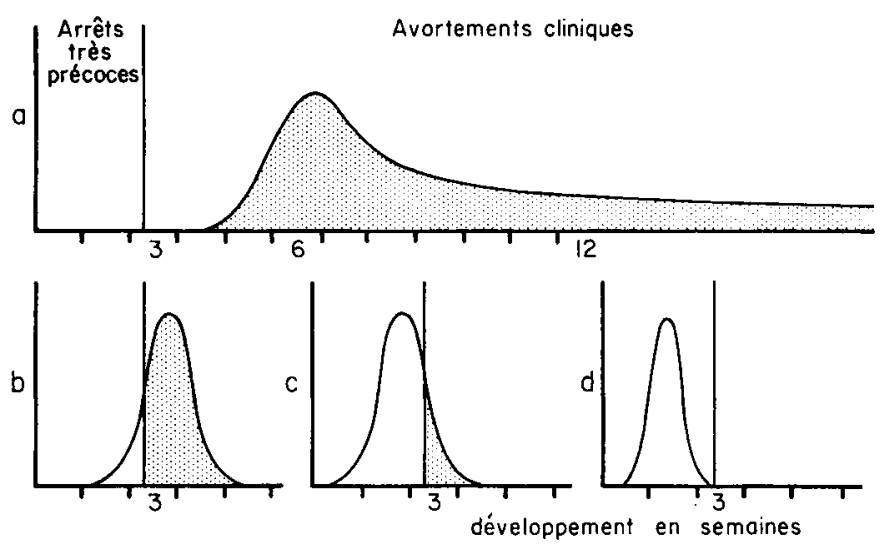

FIG. 5. - Modèles de courbes des durées du développement par rapport oux possibilités de recueil des spécimens expulsés : courbe $a$ : situation analogue à celle de la trisonie $21 ;$ courbes $b, c$ et $d$ : ces courbes montrent que les variations dans la durée du développement peuvent expliquer les fréquences observées dans les avortements spontanés sans que l'incidence à la fécondation soit différente. 
Une réserve peut être faite au sujet de la trisomie 16 dont la fréquence anormalement élevée est peut-être liée à d'autres causes (rôle de l'hétérochromatine ?).

\section{2. - Les triploïdies.}

Plusieurs erreurs chromosomiques peuvent conduire à une triploïdie : il peut s'agir de la fécondation d'un ovule haploïde (23 chromosomes) par deux spermatozoïdes haploïdes normaux : c'est la dispermie; de la fécondation d'un ovule haploïde par un spermatozoïde diploïde (46 chromosomes) : c'est la diandrie ; et enfin de la fécondation d'un ovule diploïde par un spermatozoïde haploïde : c'esil la digynie. Dans ces deux derniers mécanismes l'état diploïde du gamète peut résulter d'un accident survenu lors de la première ou lors de la deuxième division méiotique.

La répartition des chromosomes sexuels donne trois types de triploïdies : 69, $X X Y$; $69, X X X$ et $69, X Y Y$ cette dernière éventualité permet d'affirmer qu'il existe des triploïdies avec deux jeux chromosomiques paternels, mais sans qu'il soit possible de préciser le mécanisme.

D'importants progrès ont été faits dans l'analyse du mécanisme des triploïdies. Deux voies d'approche ont été utilisées :

1. La coloration des chromosomes avec une substance fluorescente, la quinacrine, met en évidence sur un même chromosome des zones de fluorescence variables selon les sujets, réalisant un véritable marquage des chromosomes à l'intérieur de la même paire.

2. Le système HLA est codé par des gènes situés sur le chromosome 6 et en déterminant les antigènes HLA chez les parents et sur les cellules des tissus d'avortements, on peut déterminer l'origine des trois chromosomes 6, donc des trois jeux chromosomiques.

Théoriquement séduisantes ces deux méthodes sont en réalité difficiles el les travaux de plusieurs groupes de recherche n'ont permis de réunir que 50 observations (tabl. 4) (Couillin et al., 1978 ; Jacobs ef al., 1978 ; Lauritsen, 1976).

TABLEAU 4

Identification du mécanisme de la triploiddie

\begin{tabular}{|c|c|c|c|c|}
\hline & & Effectif & & $\begin{array}{c}\text { p. } 100 \\
-\end{array}$ \\
\hline $\begin{array}{c}\text { Diandrie } \\
\overline{-} \\
\overline{-} \\
\text { Dispermie }\end{array}$ & 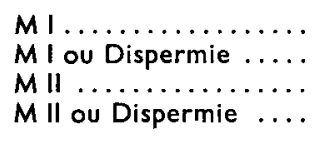 & $\begin{array}{r}0 \\
16 \\
0 \\
4 \\
18\end{array}$ & $\left.\begin{array}{r}0 \\
32 \\
0 \\
8 \\
36\end{array}\right\}$ & 76 p. 100 \\
\hline $\begin{array}{c}\text { Digynie } \\
\text { - }\end{array}$ & 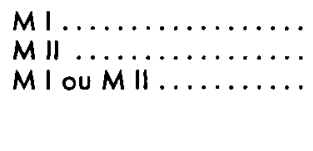 & $\begin{array}{r}9 \\
0 \\
3 \\
50\end{array}$ & $\left.\begin{array}{r}18 \\
0 \\
6\end{array}\right\}$ & 24 p. 100 \\
\hline
\end{tabular}


On constate d'abord que la digynie est le mécanisme le moins fréquent (24 p. 100). Ceci a été surprenant car dans les recherches sur divers Mammifères (Hamster, Lapin, Truie) la triploïdie était surtout le résultat de digynie. Par contre, trois fois sur quatre, on constate deux jeux chromosomiques venant du père. Dans certains cas il a été possible d'affirmer une dispermie ( 36 p. 100), mais dans d'autres cas il a été impossible de différencier diandrie et dispermie. Il semblerait par ces résultats que la dispermie est le mécanisme le plus fréquent (50 à 70 p. 100 des triploïdies).

\section{3. - Les môles hydatiformes.}

En opposition aux môles embryonnées où un caryotype triploïde est la règle, les analyses chromosomiques réalisées sur les môles hydatiformes ont régulièrement montré un caryotype diploïde mais ce caryotype est 9 fois sur $10: 46, X X$; ainsi dans notre étude sur 29 môles hydatiformes caryotypées, 25 sont $46, X X$.

Avec les techniques utilisées lors de ces recherches on était déjà frappé par une certaine homologie entre les deux chromosomes de chaque paire chromosomique. La technique de marqueurs fluorescents, déjà utilisée pour l'analyse des mécanismes des triploïdies, appliquée sur les chromosomes des cellules en culture provenant de môle et sur les chromosomes des parents, a permis à Kajii et Ohama (1977) et ensuite à Jacobs ef al. (1978) d'apporter des données fondamentales sur les mécanismes chromosomiques conduisant à une môle.

Dans toutes les observations où les marqueurs fluorescents chromosomiques étaient interprétables, les chromosomes homologues de chaque paire chromosomique étaient identiques, donc traduisant une homozygotie et de plus toujours d'origine paternelle. Il s'agit donc d'une androgenèse.

Pour expliquer cette observation on ne peut évoquer que deux mécanismes : soit fécondation par un spermatozoīde diploïde à la suite d'un accident de la deuxième division méiolique, soit fécondation par un spermatozoïde haploïde avec duplication de ses chromosomes. Dans tous les cas le jeu chromosomique de l'ovule est exclu ou inactivé. Ces deux mécanismes expliquent le caryotype 46,XX, logiquement il devrait y avoir aussi des caryotypes $46, Y Y$, mais lorsque ces accidents surviennent avec des spermatozoïdes porteurs de l'Y, l'absence des informations génétiques contenues sur le chromosome $X$ doit faire que cette éventualité est incompatible avec une prolifération cellulaire. Récemment a été rapportée une môle hydatiforme 46,XY (Surti et al., 1979) où également les deux jeux chromosomiques sont d'origine paternelle laissant supposer la fécondation par un spermatozoïde diploïde résultant d'un accident de la première division méiotique par non-réduction chromosomique, ou éventuellement une dispermie. L'ensemble de ces observations est en faveur d'un mécanisme comprenant la fécondation de l'ovule par un spermatozoïde diploïde, le jeu chromosomique maternel étant exclu.

Il s'agit donc en fait d'un mécanisme assez voisin de ce qui est rencontré dans la formation des triploïdies par diandrie. Ceci peut expliquer des observations où chez la même femme on voit successivement des avortements avec môle embryonnée et avec môle hydatiforme.

Peut-on à la lumière de ces données expliquer les phénomènes qui conduiraient à la formation des structures molaires? 

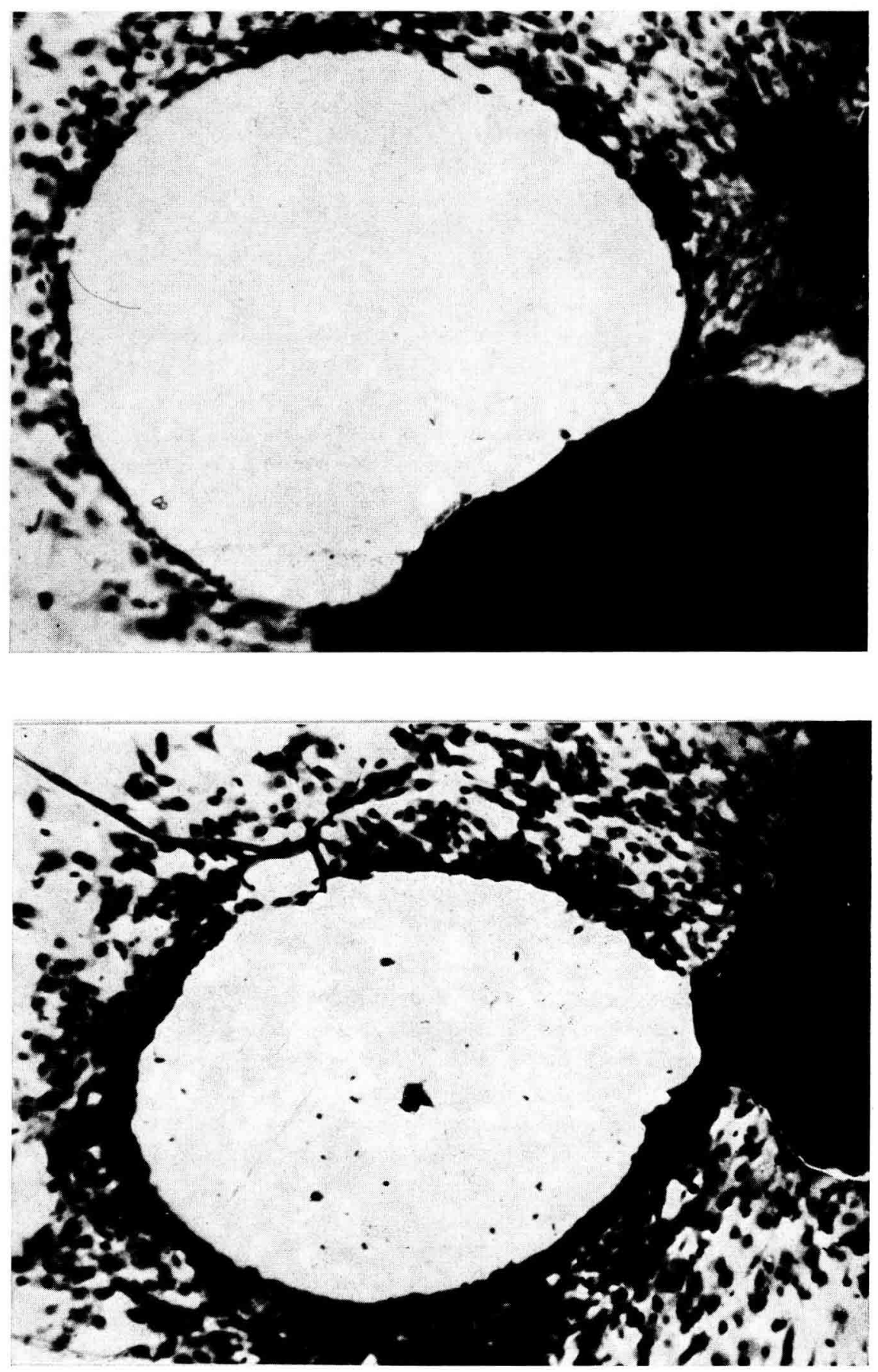

FIG. 6. - Proliférations cellulaires observées in vitro à partir d'un explant de môle hydatiforme. 
Une observation intéressante a été faite aussi bien dans notre laboratoire que dans celui de Philippe (1974) : lorsqu'on cultive in vitro des explants de môle hydatiforme on voit que, sur le verre, la prolifération cellulaire se fait par des bourgeons qui se disposent dans une structure formant un cercle qui correspond sur deux dimensions à la configuration de la môle sur trois dimensions (fig. 6). Il y a donc un phénomène de reconnaissance cellulaire qui, in vitro, conduit à la formation de cette structure.

L'analyse des antigènes HLA dans des couples ayant eu une triploïdie a montré que ces parents avaient, plus fréquemment que dans un groupe témoin, deux antigènes HLA en commun, un dans le locus A et un dans le locus B (Couillin et al., 1977).

Cette constatation est intéressante car Fellous et Dausset (1970) ont montré que les antigènes HLA sont exprimés sur les spermatozoïdes dans la zone post-acrosomiale, donc dans une région qui joue un rôle important lors de la fusion du spermatozoïde et de l'ovule. On peut se demander si une identité des antigènes HLA entre l'ovule et le spermatozoïde ne pourrait pas perturber les phénomènes de reconnaissance et permettre ainsi la pénétration d'un deuxième spermatozoïde.

Récemment un excès de parents compatibles pour deux antigènes HLA a également été retrouvé, d'une part chez des couples ayant eu des avortements répétés (Schacter et al., 1979) et d'autre part dans le cas de chorio-carcinomes (Lawler, 1978). Ces faits mériteraient des études plus poussées.

\section{4. - Les anomalies de la structure.}

Les anomalies de la structure représentent 3 à 6 p. 100 des aberrations chromosomiques observées dans les avortements. Dans notre série de 1600 avortements caryotypés (Boué ef al., 1975) nous avons 39 anomalies de la structure (tabl. 5) ; comme la plus grande partie de ce travail a été réalisée avant l'apparition des fechniques de bandes il est vraisemblable qu'un certain nombre d'anomalies de la structure ont été méconnues. Dans les translocations par fusion centrique plus de la moifié sont transmises par l'un des parents, par contre les anomalies de novo sont plus fréquentes dans les autres anomalies de la structure : délétions, trisomies partielles...

TABLEAU 5

Anomalies de la structure dans les ovorfements spontanés

\begin{tabular}{lccccc}
\hline & $\begin{array}{c}N b \\
\text { total }\end{array}$ & \multicolumn{2}{c}{ Transmises } & Mat & Pat
\end{tabular}


Il est intéressant de comparer les résultats concernant les translocations par fusion centrique trouvées dans les avortements spontanés de celles décelées lors d'un diagnostic prénatal chez des couples où l'un des parents est porteur de la translocation sous sa forme équilibrée (Boué, 1979), dans les deux cas lorsqu'un zygote porte la translocation à l'état déséquilibré celle-ci est presque toujours héritée de la mère.

On avait supposé que les différences observées dans la survenue d'anomalies déséquilibrées à la naissance, ou en diagnostic prénatal selon que la mère ou le père transmettait l'anomalie, pouvaient résulter d'une différence dans la fréquence des arrêts précoces du développement (Bové et Bové, 1975). II semble d'après les résultats sur les avortements spontanés que la rarełé de zygotes déséquilibrés quand le père transmet l'anomalie devrait plutôt résulter d'arrêts précoces méconnus ou d'une absence de conception par des spermatozoïdes déséquilibrés. Ces observations peuvent être rapprochées de ce qui est constaté avec certaines translocations par fusion centrique, d'une part chez la souris (Winking et Gropp, 1979) et d'autre part chez le bélier (Chapman et Bruère, 1975).

\section{5. - Les mosaïques.}

Alors qu'une bonne concordance est retrouvée pour les divers types d'anomalies, une divergence assez nette a été relevée pour ce qui concerne l'incidence des mosaĩques dans les avortements. Dans la plupart des séries la fréquence des mosaíques est très faible, bien que le nombre des métaphases analysées ait été suffisant pour permettre ces diagnostics, mais deux séries américaines ont rapporté une fréquence plus élevée de mosaïque, aucune explication satisfaisante n'a été donnée.

Existe-t-il d'autres causes génétiques responsables d'arrêt du développement du zygote?

Dans les avortements où un caryotype apparemment normal a été observé, l'étude anatomique a montré qu'un grand nombre (la moitié environ) présentaient des anomalies du développement semblables à celles rencontrées lorsque le caryotype est anormal, donc qu'une cause zygorique était vraisemblablement responsable de l'arrêt du développement. II est logique de penser que (de même que pour les anomalies chromosomiques) d'autres anomalies génétiques peuvent être à l'origine de certains de ces arrêts.

Dans un domaine encore mal compris sur le plan génétique, les anomalies du tube neural, des études anglaises (Creasy et Alberman, 1976) ont estimé que parmi les zygotes porteurs d'une telle anomalie, la moitié vont certainement conduire à un avortement, un quart sont une cause de mortalité périnatale et un quart conduisent à une naissance vivante. On a pu poser la question de savoir si les variations de la fréquence des anomalies du tube neural chez les enfants vivants n'étaient pas que le reflet des variations dans l'incidence des expulsions pendant la grossesse?

Une question est fréquemment posée : existe-t-il chez l'homme un ou des gènes comparables à ceux du locus $T$ chez la souris?

Une observation d'agénésie sacro-coccygienne familiale apporte des arguments en faveur de l'existence de tels gènes chez l'homme (Boué, Fellous, Malbrunot, observation non publiée). Sur quatre générations (fig. 7) on retrouve cette agénésie qui se 

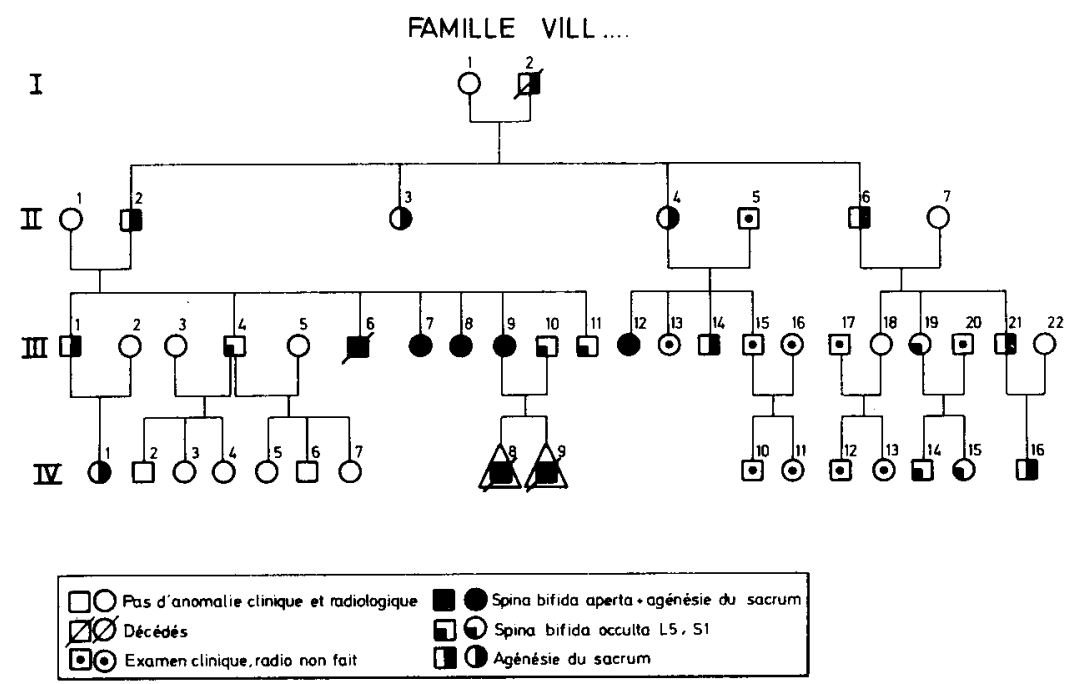

FIG. 7. - Arbre généalogique de la famille Vill. montrant la distorsion dans la ségrégation aux générations II et III et les différents degrés de gravité de l'anomalie (Boué J., Fellous M., Malbrunot C., documents inédits).

transmet sur le mode dominant, mais la ségrégation se fait de façon anormale plus de la moitié des enfants étant atteints. On remarque ensuite l'existence de degrés divers de gravité, certains sujets n'ayant qu'un spina bifida occulta, d'autres une agénésie du sacrum asymptomatique, d'autres une forme majeure d'agénésie sacrée avec spina bifida. L'éfude dans cette famille des ségrégations de l'haplotype HLA B-35 transmise par l'aïeul et l'allèle 1 du gène PGM 3 montre la même distorsion dans la ségrégation et permet de penser qu'il existe sur le chromosome 6 un «gène » responsable de cette agénésie sacro-coccygienne. Tous ces éléments sont comparables à ce qui est observé avec le locus $T$ de la souris.

Présenté au Colloque D.G.R.S.T. de Port Bail, 27 février-1 er mars 1979.

Accepté en septembre 1979.

Remerciements. - Les travaux rapportés dans ce texte ont reçu une aide financière de la DGRST (Contrats 71-7-3104, 73-7-1659) et de l'INSERM (ATP 25-75-48).

\section{Références}

BOUÉ A., 1979. European collaborative study on structural chromosome anomalies in prenatal diagnosis, 34-49. In MURKEN J. D., STENGEL-RUTKOWSKI S., SCHWINGER E., Prenatal Diagnosis, Enke Verlag, Stuttgart.

BOUÉ J., BOUÉ A., 1975. Ełudes prospectives des facteurs chromosomiques de qualité des gamètes. Ann. Biol. anim. Bioch. Biophys., 15, 753-757.

BOUÉ J., BOUÉ A., LAZAR P., 1975. Retrospective and prospective epidemiological studies of 1500 karyotyped spontaneous human abortions. Teratology, 12, 11-26. 
BOUÉ J., DAKETSÉ M. J., DELUCHAT C., RAVISÉ N., YVERT F., BOUÉ -A., 1976. Identification par les bandes $Q$ et $G$ des anomalies chromosomiques dans les avortements spontanés. Ann. Genet., 19, 233-239.

BOUE J., PHILIPPE E., GIROUD A., BOUÉ A., 1976. Phenotypic expression of lethal chromosome anomalies. Teratology, 14, 3-20.

CARR D., GEDEON M., 1978. Q-Banding of chromosomes in human spontaneous abortions. Can. J. Genet. Cytol., 20, 415-425.

CHAPMAN H. M., BRUĖRE A. N., 1975. The frequency of aneuploidy in the secondary spermatocytes of normal and robertsonian translocation-carrying rams. J. Reprod. Fert., 45, 333-342.

COUILLIN P., BOUÉ A., BOUÉ J., RAVISÉ N., FEINGOLD N., HORS J., 1977. HLA markers in parents of triploid conceptuses. Path. Biol., 25, 647-650.

COUILLIN P., HORS J., BOUÉ J., BOUÉ A., 1978. Identification of the origin of triploidy byHLA markers. Hum. Genet., 41, 35-44.

CREASY M. R., ALBERMAN E. D., 1976. Congenital malformations of the central nervous system in spontaneous abortions. J. Med. Genet., 13, 9-16.

CREASY M. R., CROLLA J. A., ALBERMAN E. D., 1976. A cytogenetic study of human spontaneous abortions using banding techriques. Hum. Genet., 31, 177-185.

DILL in CARR D., GEDEON M., 1978.

FELLOUS M., DAUSSET J., 1970. Probable haploid expression of HLA antigens on human spermatozoa. Nature, 225, 191-193.

FRENCH F. E., BIERMAN J. M., 1962. Probabilities of fetal mortality. Publ. Health Rep., 77, 835-847.

GROPP A., PUTZ B., ZIMMERMAN U., 1976. Autosomal monosomy and trisomy causing developmental failure. In GROPP A., BENIRSCHKE K., Developmental biology and pathology. Curr. Top. Pathol., 62,117 , Springer, Berlin.

GROPP A., WINKING H., PUTZ B., 1979. Critical points in development of trisomic mouse embryos. Clin. Genet. (in press).

HASSOLD T. J., MATSUYAMA A., NEWLANDS I. M., MATSURA J. S., JACOBS P. A., MANUEL B., TSUEI J., 1977. A cytogenetic study of spontaneous abortions in Hawaii. Ann. Hum. Genet., 41, 443-454.

HERTIG A. T., ROCK J. 1959. A series of potentially abortive ova recovered from fertile women prior to the first missed menstrual period. Amer. J. Obst. Gynec., 58, 968.

JACOBS P. A., ANGELL R. R., BUCHANAN I. M., HASSOLD T. J. MATSUYAMA A., MANUEL B., 1978. The origin of human triploids. Ann. Hum. Genet., 42, 49-57.

JACOBS P. A., HASSOLD T. J., MATSUYAMA AM. M., NEWLAND I. M., 1978. Chromosome constifution of gestational trophoblastic disease. Lancet, 2, 49.

KAJII T., OHAMA K., 1977. Androgenetic origin of hydatidiform mole. Nature, 268, 633-634.

KAJII T., FERRIER A., NIIKAWA N., TAKAHARA H., OHAMA K., AVIRACHAN S., 1979. Anatomic and chromosomal anomalies in 639 spontaneous abortuses. Hum. Genet (in press).

LAURITSEN J. G., 1976. Aetiology of spontaneous abortuses. Acta obsfef. gynec. scand. Supp. 52.

LAWLER S. D., 1978. HLA and trophoblastic tumors. Brit. Med. Bul., 34, 305-308.

LERIDON H., 1977. Human fertility. Univ. Chicago Press, Chicago.

PHILIPPE E., 1974. Histopathologie placentaire. Masson ed., Paris.

SCHACTER B., MUIR A., GYVES M., TASIN M., 1979. HLA-A, B compatibility in parents of offspring with neural-fube defects or couples experiencing involontary fetal wastage. Lancet, i, 796799.

SUTHERLAND G. R., CARTER R. F., BAUD R., SMITH I. I., BAIN A. D., 1978. Chromosome studies at the paediatric necropsy. Ann. Hum. Genet., 42, 173-181.

SURTI U., SZULMAN A., Q'BRIEN S., 1979. Complete (classic) hydatidiform mole with 46, XY karyotype, of paternal origin. Hum. Genet., 51, 153-155.

TAKAHARA H., in CARR D. H., GEDEON M. N., 1978.

WARBURTON D., YU C., KLINE J., STEIN Z., 1978. Mosaic autosomal trisomy in cultures from spontaneous abortions. Am. J. Hum. Genet., 30, 609-617.

WILLIAMSON E., MILLER J., 1979. A prospective study into early conceptual loss in human. Clin. Genet. (in press).

WINKING H., GROPP A., 1979. Malsegregation rates and impairment of fertility in single and multiple Robertsian metacentric heterozygous mice. Clin. Genet (in press). 